\section{Laurence Klotz}

Président, Association des urologues du Canada president@cua.org
$\mathrm{N}$ os collègues d'Edmonton ont organisé un congrès 2008 époustouflant, qui comprenait tous les ingrédients indispensables à sa réussite: de la grande science, des exposés sagaces, des débats fougueux et de merveilleuses soirées. Ce sont précisément ces éléments-là qui rendent le congrès de l'AUC si attirant. Des échanges libres et sincères, sans rancœur ni battage de marketing extravagant, se sont en effet déroulés dans une atmosphère de convivialité, lorsqu'est venu le temps d'exprimer de véritables divergences d'opinion. Nous nous devons ici de féliciter le docteur Metcalfe, ainsi que le comité scientifique et le comité organisateur local, sans oublier le personnel du Bureau central.

L'année 2008-2009 se présente comme une année de consolidation des changements fondamentaux qui façonnent l'AUC depuis les 3 dernières années. Nous avons signé une nouvelle entente contractuelle avec le Bureau central, ce qui devrait nous permettre d'assurer une imputabilité fiscale ainsi qu'une surveillance des activités quotidiennes du bureau par les cadres de l'AUC. Notre nouvelle directrice du Bureau de l'éducation, Mme Tiffany Pizioli, a reçu le mandat de rehausser l'activité et le profil du Bureau. En effet, I'AUC est agréée par le Collège royal des médecins et chirurgiens du Canada pour fournir des activités de développement professionnel continu (DPC). En tant que tel, I'AUC est donc en mesure de réviser les demandes et de fournir un certificat, selon la Section 1 ou 3 des activités éducatives telles que définies par le Collège royal. D'ailleurs, nous encourageons toute personne désireuse d'organiser une de ces activités à contacter le Bureau de l'éducation de I'AUC (tiffany.pizioli@cua.org).

En outre, nous possédons notre propre journal, le $J A \cup C$, dont les articles se retrouvent à présent sur PubMed. Si le comité de rédaction s'occupe du contenu et de la direction scientifiques, un nouveau comité d'édition, qu'il reste encore à établir et qui sera rattaché à l'exécutif de I'AUC, sera, quant à lui, en charge du côté affaires du journal.

L'AUC se réjouit d'avoir pu compter sur des chefs visionnaires et compétents depuis les 5 dernières années. Une grande partie du mérite revient à Yves Fradet et à Michael Jewett pour avoir mis en place les arrangements du Bureau central, à Luc Valiquette pour avoir eu l'initiative de démarrer le JAUC, et à Jim Metcalfe pour superviser la nouvelle alliance avec le Bureau central de la SIU.

Alors que nous entrons dans une nouvelle phase, nous sommes particulièrement heureux d'avoir à bord Denis Lavoie, comme secrétaire, et Stuart Oake, comme trésorier. Ces deux hommes sont solides comme un roc et me rendront la tâche de président beaucoup plus facile.

Le transfert d'un Bureau central doté d'une équipe de bénévoles à une équipe professionnelle, a permis à I'AUC d'atteindre une belle maturité au niveau organisationnel. Cependant, ce processus vient remettre en cause la direction et la mission de I'AUC, et I'on est d'ores et déjà en droit de se poser les questions fondamentales suivantes : Quels services I'AUC devrait-elle fournir à ses membres? Quels sont les objectifs du congrès annuel et comment devrait-on se structurer pour les atteindre? Comment arriveronsnous à relever les défis que posent les changements profonds qui commencent à surgir dans l'exercice de l'urologie? Comment le Bureau de l'éducation devrait-il se positionner face aux urologues et aux compagnies pharmaceutiques? L'AUC devrait-elle adopter un rôle plus politique à l'instar de I'AUA?

Afin d'alléger la tâche de l'Association dans la résolution de ces questions, nous avons l'intention cette année d'établir un plan stratégique à long terme, dont nous vous ferons part au fur et à mesure.

Pour terminer, je voudrais dire que c'est un grand privilège pour moi que de servir comme président de I'AUC. Pour m'épauler dans ce rôle exigeant, j'ai passé certains pouvoirs, notamment à Armen Aprikian, professeur et titulaire de la Chaire d'urologie à McGill et uro-oncologue universitaire remarquable, qui a été nommé rédacteur en chef par intérim de ce journal. Quant à John Grantmyre, professeur d'urologie à Dalhousie, il a pris la relève en tant que président par intérim du Consortium canadien sur la recherche en urologie. 